\title{
Efeitos de Instruções e do Horizonte nos Julgamentos de Tamanhos Relativos Pictóricos ${ }^{1}$
}

\author{
Nelson Torro Alves \\ Sérgio Sheiji Fukusima² \\ Universidade de São Paulo - Ribeirão Preto
}

\begin{abstract}
RESUMO - O objetivo deste trabalho foi investigar o papel das instruções de julgamento e de informações pictóricas de profundidade na percepção de tamanho relativo. No experimento, os observadores escolheram qual dentre dois retângulos (estímulo-padrão e teste) apresentados na tela de um computador era o mais alto. Os observadores foram divididos em grupos de acordo com as instruções de julgamento (aparente ou objetivo) recebidas e o tipo de gradiente de textura no qual eram apresentados os estímulos. Os julgamentos não foram afetados pela textura, mas predominantemente pelas instruções experimentais. Instruções de julgamento aparente apresentaram maiores erros relativos e favoreceram a subestimação de tamanho do estímulo-teste, enquanto instruções de julgamento objetivo favoreceram a superestimação de tamanho. A presença da linha do horizonte promoveu maior acurácia nos julgamentos de tamanho relativo. Instruções de julgamento e altura do horizonte mostraram-se, portanto, como fatores importantes na determinação dos julgamentos de tamanhos relativos percebidos visualmente.
\end{abstract}

Palavras-chave: percepção visual; instruções; informações pictóricas de profundidade.

\section{Effects of Instructions and of the Horizon on Judgments of Pictorial Relative Sizes}

\begin{abstract}
Investigating the role of the judgment instructions and pictorial depth information in the perception of relative size was the purpose of this work. In the experiment, observers chose which rectangle of the two presented (standard and test stimulus) was taller on the computer screen. The observers were divided in groups according to the instructions received (apparent or objective) and the kind of texture gradient in which the stimuli were presented. The judgments were not affected by the texture, but predominantly by the experimental instructions. Apparent judgment instructions had more relative errors and favored the underestimation of size of the standard stimulus, while objective judgment instructions favored the overestimation of size. The presence of the horizon line improved more accuracy of judgments of relative size. Judgment instructions and height of the horizon turned out as important factors in the determination of the judgments of relative size visually perceived.
\end{abstract}

Key words: visual perception; instructions; pictorial depth cues.

No campo de estudo da percepção visual tem-se freqüentemente investigado o modo como os observadores apreendem o espaço tridimensional e quais são as inter-relações envolvidas neste processo que se estabelecem entre o tamanho e a distância percebida (Gilliam, 1995). De acordo com a hipótese da invariância tamanho-distância (HITD), se mantido invariante o tamanho angular de um objeto, o tamanho percebido é diretamente proporcional à distância percebida. Esta relação pode ser ilustrada pictoricamente por dois objetos de tamanhos idênticos apresentados em um fundo com informações de profundidade, como linhas de perspectivas, em que o objeto representado mais distante parece maior que o objeto representado a distância mais próxima. Outro exemplo dessa relação perceptual entre tamanho e distância em representações pictóricas é a ilusão de Ponzo, em que duas linhas paralelas, horizontais, de mesmo tamanho e entre

1 Este trabalho é parte da monografia de conclusão de curso do primeiro autor, defendida em 2001, no Programa Optativo de Bacharelado em Psicologia. Apoio Financeiro: CNPq.

2 Endereço: Avenida Bandeirante, 3900, Ribeirão Preto, SP, Brasil 14040-901.E-mail: fukusima@ffclrp.usp.br duas linhas inclinadas convergentes, são percebidas como de tamanhos diferentes. Porém, a HITD nem sempre é evidenciada nos julgamentos de tamanho e distância, como relatado por Gogel e Da Silva (1987b). Julgamentos de tamanhos de objetos familiares, por exemplo, uma carta de baralho, não estabelecem relaçõos de direta proporcionalidade entre distâncias e tamanhos julgados, mas sim, relações de inversa proporcionalidade. Neste caso, fatores cognitivos interferem nos julgamentos de tamanho e distância, estabelecendo a chamada hipótese do paradoxo tamanho-distância, em que a constância de tamanho e memória parecem ser fundamentais para se dimensionar os julgamentos do espaço percebido visualmente (Mon-Williams \& Tresilian, 1999).

Por sua vez, a abordagem ecológica da percepção do espaço de Gibson (1979) postula que os tamanhos e distâncias relativas entre objetos são especificados pelas suas relações com o horizonte. $\mathrm{O}$ horizonte é definido como o limite mais distante no plano do solo, cuja posição é determinada pela altura do olho do observador. Deste modo, num ambiente natural, a altura de um objeto de sua base até o ponto que horizonte o intercepta corresponderá à altura do ponto de observação (nível dos olhos). Em uma representação pictórica 
(desenhos ou figuras) a altura do ponto de observação poderá não ser conhecida, entretanto, se houver dois ou mais objetos em cena o tamanho relativo dos objetos poderá ser especificado por meio de relações de razão de horizonte. A razão de horizonte corresponde à razão do tamanho total do objeto pelo tamanho parcial do objeto que se entende do plano do solo até a linha do horizonte. As análises de Sedgwick (conforme citado por Rogers, 1996) e Rogers (1996) mostram que relações de razão de horizonte representam uma poderosa fonte de informação na determinação do tamanho relativo de objetos em representações pictóricas.

A percepção de tamanho relativo pode ser determinada também por outras variáveis do arranjo óptico, tais como, as características do gradiente de textura. As distorções de tamanho observadas em algumas ilusões visuais são decorrentes das configurações do gradiente de textura que estão inseridos os estímulos (Williams \& Enns, 1996). O gradiente de textura pode afetar também a busca visual de tamanho de objetos (Aks \& Enns, 1996).

Além disso, o julgamento do tamanho absoluto de objetos pode ser influenciado pelo tipo de atitude de julgamento do observador frente ao estímulo visual. A atitude de julgamento objetivo toma como parâmetro o tamanho real do objeto, enquanto a atitude de julgamento aparente tem como referência o tamanho aparente do objeto, isto é, o tamanho que ele aparenta possuir (Predebon, 1992). Alguns trabalhos (Fukusima \& Da Silva, 1999; Predebon, 1992) mostram que sob condições de reduzida estimulação visual as instruções de julgamento influenciam as estimativas de tamanho absoluto de objetos. Entretanto, ainda não existem trabalhos que tenham investigado sistematicamente o papel das instruções de julgamento (aparente e objetivo) na avaliação do tamanho relativo em representações pictóricas.

O presente estudo teve por objetivo, portanto, investigar como instruções de julgamento (aparente e objetivo) afetam as estimativas do tamanho relativo em representações pictóricas. No experimento realizado, foram também investigados os efeitos de três diferentes gradientes de textura e da altura da linha do horizonte nos julgamentos de tamanho relativo dos observadores.

\section{Método}

\section{Participantes}

Participaram do estudo 49 universitários (19M, 30F) do campus da Universidade de São Paulo em Ribeirão Preto, com idade entre 17 e 33 anos e acuidade visual normal (6/6) ou superior em ambos os olhos com ou sem lentes corretivas. Os participantes foram distribuídos aleatoriamente em sete grupos eqüitativos. Todos os participantes assinaram o termo de consentimento aprovado pelo Comitê de Ética da FFCLRP e receberam pequena ajuda de custo para ressarcir eventuais despesas decorrentes da participação neste experimento.

\section{Material e equipamento}

Um aparelho Orthorather da Bausch \& Lomb foi usado para medir a acuidade visual dos participantes. Um apoio para o queixo foi utilizado para posicionar adequadamente a cabe- ça do participante frente a um monitor Philips Brilliance 21A conectado a um computador 486DX2, 50MHz, 4MbRAM, com placa de vídeo Diamond SpeedStar24 de 1MbRAM.

Um programa desenvolvido em Turbo Pascal 7.0 foi utilizado para gerar e apresentar os estímulos na tela do computador e para coletar as respostas dos participantes. Os estímulos consistiam de dois retângulos brancos apresentados simultaneamente, um na parte superior (estímulo-teste) e outro na parte inferior (estímulo-padrão). O estímulo-padrão podia medir 5,40 x 3,48 cm (Figuras $1 \mathrm{~A}$ e 1B) ou 3,60 x 2,32 cm (Figuras 1C e $1 \mathrm{D})$. O tamanho inicial do estímulo-teste podia ser equivalente a $130 \%$ ou $70 \%$ do estímulo-padrão. Os dois retângulos eram apresentados na tela do computador juntamente a uma das quatro diferentes condições de textura do experimento: (1) sem textura (figura 1A), (2) textura de compressão de linhas horizontais (Figura 1B), (3) textura de linhas de perspectiva (Figura 1C) e (4) textura composta pela sobreposição das texturas de linhas de perspectiva e compressão de linhas horizontais (Figura 1D). Além disso, para cada uma das condições de textura foram elaboradas duas diferentes condições de horizonte. Na primeira condição, não havia representação da linha do horizonte (Figuras 1A e 1C) e, na segunda condição, a linha do horizonte era apresentada acima do ponto de fixação (Figuras 1B e 1D). A distância entre a linha do horizonte e o ponto de fixação correspondia a $30 \%$ da altura total da tela.

\section{Procedimento}

Em sessão individual, o participante (com o olho esquerdo vendado) sentou-se em frente ao computador e posicionou sua cabeça sobre um apoio para o queixo de forma que o seu olho direito ficasse a $50 \mathrm{~cm}$ de distância do centro da tela do computador. Por meio do método das escadas duplas foram coletadas as respostas dos observadores. O ajustamento de tamanho do estímulo-teste de uma apresentação de estímulos a outra foi feita em incrementos ou decrementos de $2 \%$
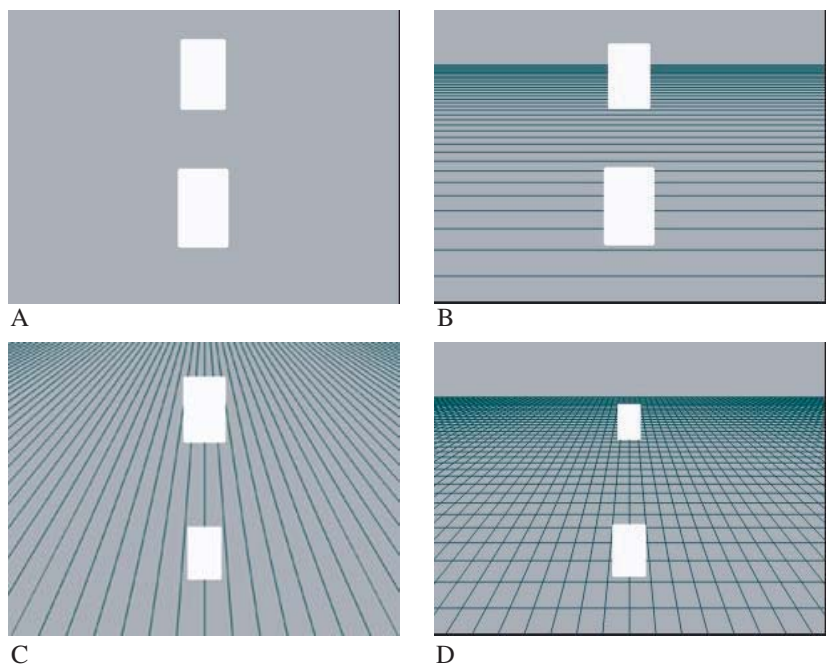

$\mathrm{D}$

Figura 1. Exemplos de estímulos.

Nota: a) ausência de textura e ausência do horizonte. b) gradiente de compressão e presença do horizonte. c) gradiente de perspectiva e ausência do horizonte. d) gradiente de compressão e perspectiva e presença do horizonte 
$(0,11 \mathrm{~cm}$ para largura e $0,07 \mathrm{~cm}$ para altura) do tamanho do estímulo-padrão (5,4 x 3,48 ou 3,6 x 2,32 cm). Para isto, a tarefa dos observadores era julgar qual estímulo era maior. Se os observadores julgassem o estímulo-teste como maior que o estímulo-padrão, pressionaria a tecla "A", e o tamanho do estímulo-teste seria diminuído na próxima apresentação em $2 \%$. Se os observadores, por outro lado, julgasse o estímulo-padrão maior que o estímulo-teste, pressionaria a tecla "D", e o tamanho do estímulo-teste seria reduzido em $2 \%$ na próxima apresentação.

O delineamento experimental é indicado na Quadro 1, onde se mostra a distribuição dos grupos de observadores em cada condição de instrução e gradiente de textura. Observadores dos grupos 1,3 e 5 receberam instruções que enfatizavam estratégias de julgamento com base no tamanho objetivo das figuras e os observadores dos grupos 2, 4 e 6 instruções que enfatizavam estratégias de julgamento com base no tamanho aparente das figuras. O grupo 7 (controle) não recebeu instruções específicas de julgamento.

O julgamento objetivo é baseado na estimativa do tamanho real do objeto; neste caso o gradiente de textura ao fundo que estiver presente durante a apresentação do estímulo deve ser desconsiderado. No julgamento aparente, a estimativa é realizada tentando-se "inserir" os estímulos na textura que induz a noção de profundidade (vide Anexo). Quatro condições de textura foram utilizadas no experimento. Os grupos 1 e 2 julgaram os tamanhos dos estímulos sobre gradiente de compressão de linhas horizontais (Figura 1B); os grupos 3 e 4 sobre gradiente linhas de perspectiva (Figura 1C); os grupos 5 e 6 sobre gradiente combinado de compressão de linhas horizontais e de perspectiva (Figura 1D) e o grupo 7 em condição de ausência de textura (Figura 1A).

Cada sessão experimental foi composta por quatro blocos de 50 apresentações de estímulos. Os blocos diferenciavam entre si com relação à presença ou ausência da linha do horizonte e tamanho do estímulo-padrão (pequeno ou grande). Todos os observadores foram submetidos a estas quatro condições de estímulo.

Quadro 1. Delineamento experimental

\begin{tabular}{lccc}
\hline Textura & $\begin{array}{c}\text { Instruções de } \\
\text { julgamento } \\
\text { objetivo }\end{array}$ & $\begin{array}{c}\text { Instruções de } \\
\text { julgamento } \\
\text { aparente }\end{array}$ & $\begin{array}{c}\text { Ausência de } \\
\text { instruções }\end{array}$ \\
\hline Compressão & Grupo 1 & Grupo 2 & -------- \\
\hline Perspectiva & Grupo 3 & Grupo 4 & -------- \\
\hline $\begin{array}{l}\text { Compressão e } \\
\text { perspectiva }\end{array}$ & Grupo 5 & Grupo 6 & -------- \\
\hline $\begin{array}{l}\text { Ausência de } \\
\text { textura }\end{array}$ & ------- & ------ & Grupo 7 \\
\hline
\end{tabular}

\section{Análise dos dados}

A cada observador, as médias dos tamanhos ajustados do estímulo-teste nas últimas apresentações de cada bloco, que tenderam a convergir e a se estabilizar em torno de um tamanho específico, foram tomadas como uma estimativa do ponto de igualdade subjetiva (PIS), que logo em seguida foi utilizada para se determinar o erro relativo pela seguinte fórmula:
Erro $(\%)=100 *($ EP-PIS $) / E P$, onde Erro $(\%)$ é o erro relativo e EP é o tamanho do estímulo-padrão.

Sob instrução objetiva, os valores de EP foram $3,60 \mathrm{~cm}$ e $5,40 \mathrm{~cm}$, que correspondiam respectivamente às alturas físicas do retângulo pequeno e grande. Sob instrução aparente, esses valores foram $1,50 \mathrm{~cm}$ e $2,24 \mathrm{~cm}$, que correspondiam respectivamente às alturas do retângulo pequeno e grande determinadas pelos ângulos visuais, se eles fossem representados na tela do computador a mesma distância do estímulo-teste.

Erro relativo negativo indica que o estímulo-teste foi ajustado maior que o estímulo-padrão, ou seja, o estímulo-teste foi subestimado perceptivamente em relação ao padrão. Erro positivo indica que o estímulo-teste foi ajustado menor que o padrão, ou seja, o teste foi superestimado perceptivelmente em relação ao estímulo-padrão.

\section{Resultados}

Os erros relativos foram submetidos inicialmente a uma análise de variância (ANOVA) modelo between-within: [sete grupos de observadores x (2 tamanhos de retângulos x 2 condições de horizonte)]. Esta ANOVA indicou efeitos significativos dos fatores: tamanho do retângulo (pequeno ou grande) $[F(1,42)=1617,41 ; p \leq 0,001]$, condição de horizonte (presença ou ausência) $[F(1,42)=73,26 ; p \leq 0,001] \mathrm{e}$ grupo de observadores $[F(6,42)=7,73 ; p \leq 0,001]$ sobre os erros relativos ao se julgar os tamanhos do retângulo branco. Entretanto, nesta análise inicial, não foi possível identificar se a diferença entre grupo de observadores do experimento havia sido decorrente do tipo de instruções que receberam os observadores ou do gradiente de textura utilizado. Com a finalidade de se separar estes efeitos realizou-se uma ANOVA between-within adicional da qual foram excluídos os resultados do grupo controle (ausência de textura), cujo modelo foi: [ 2 instruções x 3 gradientes de texturas x (2 tamanhos de retângulos x 2 condições de horizonte)]. Podemos, então, verificar que as diferenças entre os grupos estavam sendo determinadas pelas instruções de julgamento que receberam os observadores no experimento $[F(1,6)=355,173 ; p \leq 0,001]$ e não pelo gradiente de textura utilizado $[F(2,36)=0,067$; $p \geq 0,1]$. Os três diferentes gradientes de textura elaborados para o experimento: 1) Gradiente de compressão de linhas horizontais, 2) Gradiente de linhas de perspectiva e 3) Gradiente combinado por compressão de linhas horizontais e linhas de perspectiva, afetaram da mesma maneira os julgamentos de tamanho relativo dos observadores. Instruções de julgamento aparente e de julgamento objetivo, entretanto, tiveram efeitos distintos sobre os julgamentos de tamanho relativo dos observadores. O uso de instruções de julgamento aparente favoreceu a subestimação de tamanho do retângulo superior (estímulo-teste) em relação ao retângulo inferior (estímulo-padrão), sendo o retângulo localizado na parte superior da tela ajustado para um tamanho maior que aquele que deveria na realidade possuir. Instruções de julgamento objetivo, por outro lado, promoveram a superestimação do estímulo-teste, que foi ajustado, conseqüentemente, como menor que o estímulo-padrão. Houve também uma diferença significativa entre os erros percentuais para instruções de julgamento objetivo e aparente. $[F(2,12)=501,31$; $p \leq 0,001]$. Maiores erros relativos foram obtidos, em todas as 


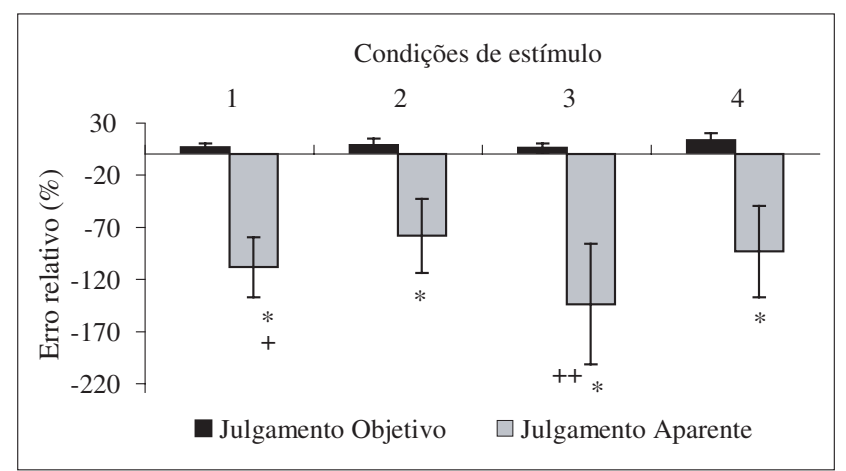

Figura 2. Efeito das instruções e do horizonte sobre os julgamentos de tamanho relativo para os gradientes de compressão, perspectiva e combinado. Nota: As condições de estímulo são: 1) Ausência do horizonte e tamanho grande do EP, 2) Presença do horizonte e tamanho grande do EP, 3) Ausência do horizonte e tamanho pequeno do EP e 4) Presença do horizonte e tamanho pequeno do EP.

* $\quad(p<0,001)$ julgamento aparente comparado com objetivo

$+\quad(p<0,05)$ condição de estímulo 1 comparada com 2

$++(p<0,05)$ condição de estímulo 3 comparada com 4

condições de estímulo, quando os observadores eram orientados a realizarem seus julgamentos com base no tamanho aparente dos retângulos (Figura 2).

Diversos trabalhos têm mostrado que o tipo de instrução experimental utilizada afeta os julgamentos do tamanho absoluto de objetos. Predebon (1992) mostrou que os valores das estimativas, em condições de reduzida estimulação visual, do tamanho de cartas de baralho e de retângulos brancos são maiores sob instruções de julgamento objetivo que sob julgamento aparente. De modo semelhante, Da Silva e Fukusima (1999) observaram o efeito das instruções nas estimativas do tamanho absoluto de objetos familiares e não familiares. No experimento realizado, as estimativas de tamanho sob instruções aparentes foram menores que sob instruções objetivas. A teoria da percepção de tamanhos anormais (Gogel \& Da Silva, 1987a) postula que instruções de julgamento objetivo, em comparação com instruções aparentes, são mais propícias a fazer com que os observadores baseiem seus julgamentos em fontes cognitivas e não perceptuais de informação espacial. Os resultados do presente experimento mostram, portanto, que instruções que orientam atitudes perceptivas e/ou cognitivas dos observadores nos julgamentos desempenham um papel importante não apenas na avaliação do tamanho absoluto em condições de reduzida estimulação visual, mas também do tamanho relativo em representações pictóricas.

Foi também observado um efeito significativo da condição de horizonte utilizada sobre as estimativas de tamanho relativo dos observadores $[F(2,12)=153,73 ; p \leq 0,001]$. Para os dois tamanhos de estímulo-padrão (pequeno e grande), sob instruções de julgamento aparente, menores erros relativos foram cometidos quando a linha do horizonte encontrava-se presente (Figura 2). Quando utilizadas instruções de julgamento objetivo, para o tamanho pequeno do estimulo-padrão, menores erros relativos foram cometidos quando a linha do horizonte encontrava-se presente. Podemos, portanto, verificar nesta análise que a presença da linha do horizonte foi um fator promotor da acurácia nos julgamentos de tamanho relativo dos observadores. Rogers (1996) mostrou que a relação de razão de horizonte pode ser uma fonte efetiva de informação para tamanho relativo em figuras sob duas condições: 1) Quando a distância entre os estímulos na figura não é muito grande, e 2) Quando a linha do horizonte não é apresentada nem muito alta e nem muito baixo na figura; isto, possivelmente, devido ao fato dos observadores não identificarem a linha horizontal como o horizonte mas apenas como a borda da figura apresentada. Deste modo, podemos supor no presente experimento que a linha do horizonte simulada para os três gradientes de textura foi realmente considerada pelos observadores como o horizonte da representação pictórica, visto seu papel determinante no aumento da acurácia dos julgamentos de tamanho relativo. No grupo controle, entretanto, caracterizado pela ausência do gradiente de textura, a linha do horizonte não afetou as estimativas do tamanho relativo. Podemos considerar, deste modo, que o gradiente de textura fornece indícios que possibilitem ao observador considerar a linha do horizonte simulada como sendo, de fato, a linha do horizonte da figura e não apenas a sua borda. Quando esta informação é detectada, o espaço pictórico pode ser processado de modo semelhante ao espaço real, e o observador, conseqüentemente, utiliza o princípio da relação de razão de horizonte nos julgamentos de tamanho relativo, aumentando a acurácia das estimativas.

Foi observada uma interação significativa entre tipo de instrução e tamanho do estímulo-padrão sobre os erros relativos de tamanho $[F(3,18)=35,41 ; p \leq 0,001]$. Sob instruções de julgamento aparente, menores erros relativos foram obtidos nas estimativas de tamanho relativo do estímulo-padrão de tamanho grande. Para instruções de julgamento objetivo, entretanto, não houve diferenças entre os erros relativos das estimativas de tamanho dos estímulos-padrão de tamanho grande e pequeno.

\section{Discussão}

Na ANOVA realizada inicialmente não foi possível determinar se o efeito da condição grupo de observadores era resultante do gradiente de textura utilizado ou das instruções de julgamento (aparente ou objetiva). Por esta razão, uma segunda análise estatística foi realizada, retirando-se os julgamentos dos observadores do grupo na condição "ausência de textura". Surpreendentemente, a análise revelou que os julgamentos de tamanho relativo não foram afetados pelo gradiente de textura utilizado, mas preponderantemente pelas instruções que receberam os observadores. Instruções de julgamento objetivo favoreceram a superestimação do tamanho do estímulo-teste, enquanto instruções de julgamento aparente favoreceram a subestimação de seu tamanho. É interessante observar a grande diferença existente entre erros percentuais na avaliação do tamanho relativo cometidos sob instruções de julgamento aparente e objetivo. Os erros são consideravelmente maiores quando os observadores são orientados a utilizar atitudes de julgamento aparente. Alguns trabalhos envolvendo o processamento da visão em ambientes reais, ainda que sob condições de reduzida estimulação visual, mostram que as estimativas do tamanho absoluto de objetos variam de acordo com as instruções experimentais (objetivo ou aparente) (Predebon, 1992). Entretanto, os resultados do presente experimento mostram que as diferenças entre os 
dois tipos de instrução parecem ainda maiores quando os observadores são requisitados a avaliar o tamanho relativo de objetos representados graficamente. Podemos constatar no presente trabalho, portanto, que as instruções de julgamento aparente e objetivo desempenham um papel importante não apenas na determinação do tamanho absoluto, mas também do tamanho relativo.

Foi também verificado que a altura do horizonte na figura teve grande influência sobre os julgamentos de tamanho relativo. Os observadores realizaram estimativas mais acuradas dos tamanhos relativos dos retângulos na condição: "presença do horizonte". Nesta condição, a linha do horizonte situavase um pouco abaixo no plano figura, ficando mais próxima, consequientemente, dos estímulos que deveriam ser comparados (retângulos brancos) e também da altura do nível dos olhos do observador. A melhor acurácia dos observadores nesta condição de horizonte pode estar relacionada a dois fatores principais: 1) Quando os retângulos estão situados mais próximos à linha do horizonte a relação de razão de horizonte pode ser utilizada pelo observador, o que promove a acurácia de seus julgamentos; 2) Visto que a percepção de tamanho relativo é melhor ao nível dos olhos (Bertamini, Yang \& Proffitt, 1998), nessa condição de horizonte, a altura da linha do horizonte se aproxima da altura dos olhos do observador e, conseqüentemente, também promove o aumento da acurácia dos julgamentos. Existem na literatura evidências experimentais que atestam a importância destes dois fatores (Bertamini \& cols., 1998; Rogers, 1996); assim, podemos considerar que ambos influenciam os julgamentos de tamanho relativo e atuam de forma a promover sua acurácia. Estes resultados são consistentes com a abordagem ecológica da percepção visual de Gibson (1979).

Em resumo, podemos constatar no presente trabalho que a presença do horizonte melhora as estimativas de tamanho relativo de objetos representados de maneira pictórica e que as instruções são um importante fator na determinação dos julgamentos de tamanho relativo. Estudos que se proponham a investigar a percepção de tamanho relativo em representação gráficas devem, portanto, considerar para o papel essencial que as instruções (aparente ou objetivo) desempenham nos julgamentos dos observadores, e que, senão devidamente controladas, podem certamente conduzir a diferentes resultados experimentais.

\section{Referências}

Aks, D. J. \& Enns, J. T. (1996). Visual search for size is influenced by a background texture gradient. Journal of Experimental Psychology: Human Perception and Performance, 22(6), 1467-1481.

Bertamini, M., Yang, T. L. \& Proffitt, D. R. (1998). Relative size perception at a distance is best at eye level. Perception \& Psychophysics, 60(4), 673-682.

Fukusima, S. S. \& Da Silva, J. A. (1999). Familiar size as a cue to size and distance estimates. Em P. R. Killen \& W. R. Uttal (Orgs.), Fechner Day 99 (pp.198-203). Tempe: International Society for Psychophysics.

Gibson, J. J. (1979). The ecological approach to visual perception. Boston: Houghton Mifflin.

Gilliam, B. (1995). The perception of special layout from static optical information. Em W. Epstein \& S. Rogers (Orgs.), Handbook of perception and cognition: Perception of space and motion. (pp. 23-67). London: Academic Press.

Gogel, W. C. \& Da Silva, J. A. (1987a). A two-process theory of the response to size and distance. Perception \& Psychophysics, 41(3), 220-238.

Gogel, W. C. \& Da Silva, J. A. (1987b). Familiar size and the theory of off-sized perceptions. Perception \& Psychophysics, 41(4), 318-328.

Mon-Williams, M. \& Tresilian, J. R. (1999). The size-distance paradox is a cognitive phenomenon. Experimental Brain Research, 126(4), 578-582.

Predebon, J. (1992). The role of instructions and familiar size in absolute judgments of size and distance. Perception \& Psychophysics, 51(4), 344-354.

Rogers, S. (1996). The horizon-ratio relation as information for relative size in pictures. Perception \& Psychophysics, 58(1), 142-152.

William, P. A. \& Enns, J. T. (1996). Pictorial depth and framing have independent effects on the horizontal-vertical illusion. Perception, 25(8), 921- 926.

Recebido em 14.05.2004

Primeira decisão editorial em 15.07.2005

Versão final em 27.07.2005

Aceito em 05.08.2005 


\section{Anexo}

\section{Instruções de julgamento objetivo}

Serão apresentadas na tela do computador dois retângulos brancos sobre diferentes condições de textura que induzem a sensação de profundidade. Você deverá comparar a altura dos dois retângulos apresentados e identificar qual deles é o mais alto. Se você considerar que o retângulo localizado acima possui a maior altura você deverá pressionar a letra "A" no teclado. Se, ao contrário, você achar que o retângulo localizado abaixo aparenta possuir a maior altura você deverá pressionar a letra "D". Nos julgamentos de tamanho tente desconsiderar a largura dos retângulos, se concentrando somente em sua altura. Ao comparar a altura dos retângulos você deverá desconsiderar completamente o gradiente de textura que é apresentado ao fundo. Para comparar a altura imagine que você estivesse pegando uma régua e comparando o tamanho real dos retângulos na tela do computador. Utilize este critério para realizar os julgamentos, tentado desconsiderar as texturas e linhas do horizonte que são apresentadas ao fundo.

\section{Instruções de julgamento aparente}

Serão apresentadas na tela do computador dois retângulos brancos sobre diferentes condições de textura que induzem a sensação de profundidade. Você deverá comparar a altura dos dois retângulos apresentados e identificar qual deles é o mais alto. Se você considerar que o retângulo localizado acima possui a maior altura você deverá pressionar a letra "A" no teclado. Se, ao contrário, você achar que o retângulo localizado abaixo aparenta possuir a maior altura você deverá pressionar a letra "D". Nos julgamentos de tamanho tente desconsiderar a largura dos retângulos, se concentrando somente em sua altura. Ao comparar a altura dos retângulos você deverá imaginar que os retângulos estão inseridos no gradiente de textura que induz a sensação de profundidade. Avalie a altura dos retângulos com base no tamanho que eles deveriam ter se estivessem imersos na figura em profundidade. Utilize este critério para realizar os julgamentos, tentando sempre considerar as texturas e linhas do horizonte que são apresentadas ao fundo. 